October 2019

\title{
Non-relativistic susceptibility and a dark matter application
}

\author{
S. Biondini ${ }^{\mathrm{a}}$, Seyong Kim ${ }^{\mathrm{b}, \mathrm{c}}$ and M. Laine ${ }^{\mathrm{c}}$ \\ ${ }^{a}$ Van Swinderen Institute, University of Groningen, \\ Nijenborgh 4, NL-9747 AG Groningen, the Netherlands \\ ${ }^{\mathrm{b}}$ Department of Physics, Sejong University, Seoul 143-747, South Korea \\ ${ }^{\mathrm{c}} A E C$, Institute for Theoretical Physics, University of Bern, \\ Sidlerstrasse 5, CH-3012 Bern, Switzerland
}

\begin{abstract}
When thermal rate equations are derived for the evolution of slow variables, it is often practical to parametrize the right-hand side with chemical potentials. To close the system, the chemical potentials are subsequently re-expressed in terms of the slow variables, which involves the consideration of a "susceptibility". Here we study a non-relativistic situation in which chemical potentials are large compared with the temperature, as is relevant for late-time pair annihilations in dark matter freeze-out. An order-of-magnitude estimate and a lattice simulation are presented for a susceptibility dominated by bound states of stoplike mediators. After this "calibration", the formalism is applied to a model with Majorana singlet dark matter, confirming that masses up to the multi-TeV domain are viable in the presence of sufficient (though not beyond a limit) mass degeneracy in the dark sector.
\end{abstract}




\section{Introduction}

In the weakly interacting massive particle (WIMP) scenario, the number density of the dark sector is usually assumed to satisfy the so-called Lee-Weinberg equation [1],

$$
\dot{n}+3 H n=-\langle\sigma v\rangle\left(n^{2}-n_{\mathrm{eq}}^{2}\right),
$$

where $H$ is the Hubble rate. Eq. (1.1) can be derived from Boltzmann equations, assuming kinetic equilibrium and integrating over momenta [2,3]. However, Boltzmann equations have a limited range of validity, failing e.g. if interactions within the dark sector become strong.

If the interactions are strong enough to form bound states, a standard practice is to add bound states as additional degrees of freedom in a set of Boltzmann equations [4,5]. However, there are challenges with this approach. One problem is that strongly interacting systems have many bound states; another is that their number varies with the temperature [6]; a further one is that bound-state rate coefficients are cumbersome to compute. It would be attractive to have a more "inclusive" framework which does not require a priori knowledge of how many (if any) bound states are present, even if at very low temperatures a set of coupled equations surely becomes necessary.

One way to promote eq. (1.1) beyond Boltzmann equations is to note that the coefficient $\langle\sigma v\rangle$ is independent of the value of the dynamical variable $n$. Thus, one can assume that the system is prepared in a state close to equilibrium, and linearize in deviations. Thereby we can make contact with linear response theory, which permits to define a chemical equilibration rate, $\Gamma_{\text {chem }}=2 n_{\text {eq }}\langle\sigma v\rangle$, on a non-perturbative level [7]. Furthermore, within the non-relativistic expansion [8], $\Gamma_{\text {chem }}$ can be related to the thermal expectation value of a local annihilation operator, and then be measured with lattice simulations if necessary [9].

Another generalization of eq. (1.1) was put forward in ref. [10]. Making use of SchwingerKeldysh formalism, which goes beyond linear response theory, the authors reproduced the expression of ref. 9] for $\langle\sigma v\rangle$, but in addition suggested that the functional form should read

$$
\dot{n}+3 H n=-\langle\sigma v\rangle\left(e^{2 \beta \mu(n)}-1\right) n_{\mathrm{eq}}^{2},
$$

where $\beta \equiv 1 / T$ and $\mu$ couples to the total number of dark sector particles. In a weakly

coupled system, $e^{\beta \mu} n_{\text {eq }} \approx n$ (cf. eq. (2.12) ), but in general this need not be the case. The relation between $n$ and $\mu$ leads to a variant of the Saha equation, familiar from the physics of recombination, displaying significant modifications if $T \lesssim \Delta E$, where $\Delta E$ is a binding energy.

In general, the quantity $\partial n / \partial \mu$ is called a "susceptibility". In many cosmological problems, such as leptogenesis, we find ourselves in the regime $\mu \ll T$; susceptibilities for this situation have been worked out up to higher perturbative orders [11,12]. For WIMPs, it is the nonrelativistic regime $\mu \sim M \gg T$ that needs to be attacked. The goal of the present study is to define and estimate a susceptibility for the latter situation, and to show how the corresponding result can be implemented in a dark matter computation employing eq. (1.2). 


\section{General setup}

We consider a theory whose dark sector contains a charged field, whose quanta may be called particles and antiparticles. This charged field plays the role of a "mediator", i.e. it couples dark matter to Standard Model particles. If the coupling goes through Yukawa interactions, the mediator has the charge assignment of one of the Standard Model fields, for instance that of a right-handed top quark. We assume that the mediator interacts strongly through $\mathrm{SU}\left(N_{\mathrm{c}}\right)$ gauge theory. Its gauge coupling is denoted by $g^{2}$, the Casimir coefficient of the fundamental representation by $C_{\mathrm{F}} \equiv\left(N_{\mathrm{c}}^{2}-1\right) /\left(2 N_{\mathrm{c}}\right)$, and we let $\alpha \equiv g^{2} C_{\mathrm{F}} /(4 \pi)$.

Let $\hat{\theta}$ and $\hat{\eta}$ be field operators which annihilate particles and antiparticles of the charged field, respectively, and define the number density operator by

$$
\hat{N}=\int_{\mathbf{x}} \hat{n}(\mathbf{x}), \quad \hat{n}(\mathbf{x}) \equiv \hat{\theta}^{\dagger} \hat{\theta}+\hat{\eta}^{\dagger} \hat{\eta} .
$$

Moreover we denote by $n_{\text {eq }}$ the expectation value of $\hat{n}$ in full chemical equilibrium, i.e.

$$
n_{\mathrm{eq}} \equiv \lim _{\mu \rightarrow 0}\langle\hat{n}\rangle \text {. }
$$

The role of $\mu$ is defined through eq. (2.3). We assume that $\theta$ and $\eta$ have $d_{s} N_{\mathrm{c}}$ real components $\left(N_{\mathrm{c}} \equiv 3\right)$, where $d_{s} \equiv 2 s+1$ is the degeneracy of spin degrees of freedom.

Because the processes which change the number density are very slow 1 it is appropriate to consider a state of the system in which $n \neq n_{\text {eq }}$. This can be imposed by coupling $\hat{N}$ to a chemical potential, so that the density matrix has the form

$$
\hat{\rho} \equiv \frac{\exp [-\beta(\hat{H}-\mu \hat{N})]}{Z}
$$

where the partition function is given by $Z=\operatorname{Tr} e^{-\beta(\hat{H}-\mu \hat{N})}$. In the thermodynamic limit the partition function can be parametrized by the pressure $p$ as $Z=e^{p \beta V}$, where $V$ is the spatial volume. The number density is obtained as

$$
n(\mu)=\frac{\partial p}{\partial \mu}=\frac{\langle\hat{N}\rangle}{V}=\langle\hat{n}(\mathbf{0})\rangle, \quad\langle\ldots\rangle \equiv \operatorname{Tr}[\hat{\rho}(\ldots)],
$$

where we assumed the system to be translationally invariant. A susceptibility is defined as

$$
\chi \equiv T \frac{\partial n}{\partial \mu}=\frac{\left\langle\hat{N}^{2}\right\rangle-\langle\hat{N}\rangle^{2}}{V}=\int_{\mathbf{x}}\left\{\langle\hat{n}(\mathbf{x}) \hat{n}(\mathbf{0})\rangle-\langle\hat{n}(\mathbf{0})\rangle^{2}\right\} .
$$

We now formally expand the pressure in a fugacity expansion,

$$
p=p_{0}+p_{1} e^{\beta \mu}+p_{2} e^{2 \beta \mu}+\ldots,
$$

\footnotetext{
${ }^{1}$ By slow we mean slow compared with processes responsible for kinetic equilibration, and with reactions between Standard Model particles; that is, the number density is assumed to be the only non-equilibrium variable.
} 
where $p_{n} \sim e^{-n M / T}$ (cf. eq. (3.2)) and $M$ is the dark matter mass scale. Let us assume that the coefficient $p_{2}$ could be anomalously large because of a bound-state contribution. From eqs. (2.4), (2.5), the corresponding expansions for $n$ and $\chi$ read

$$
\begin{aligned}
& n T=p_{1} e^{\beta \mu}+2 p_{2} e^{2 \beta \mu}+\ldots, \\
& \chi T=p_{1} e^{\beta \mu}+4 p_{2} e^{2 \beta \mu}+\ldots .
\end{aligned}
$$

From eq. (2.7), omitting $p_{3}$ and higher-order terms 2 we get, in accordance with ref. [10,

$$
e^{\beta \mu} \approx \frac{-p_{1}+\sqrt{p_{1}^{2}+8 p_{2} n T}}{4 p_{2}} .
$$

Moreover, by subtracting eq. (2.8) from (2.7), we can estimate the coefficient $p_{2}$ as

$$
2 p_{2} e^{2 \beta \mu} \approx T(\chi-n) .
$$

In practical applications, it is convenient to remove exponentially small terms by noting that in the limit of chemical equilibrium, when effects suppressed by $e^{-M / T}$ can be omitted, we can identify $p_{1}=n_{\text {eq }} T$ (cf. eq. (2.7) $)$. Moreover we can define

$$
p_{2} \equiv \hat{p}_{2} n_{\mathrm{eq}}^{2} T
$$

Then the combination appearing in eq. (1.2) becomes

$$
e^{\beta \mu} n_{\mathrm{eq}} \approx \frac{-1+\sqrt{1+8 \hat{p}_{2} n}}{4 \hat{p}_{2}}=\frac{2 n}{1+\sqrt{1+8 \hat{p}_{2} n}} .
$$

In perturbation theory, $\hat{p}_{2}$ is generated by interactions. In a weakly coupled system, we may expect it to be small, in which limit eq. (2.12) reduces to $e^{\beta \mu} n_{\text {eq }} \approx n$.

\section{Order-of-magnitude estimate}

In order to estimate the magnitude of $\hat{p}_{2}$, it is useful to employ the canonical formalism. Let us denote the eigenstates of the Hamiltonian by

$$
\left|n_{\theta}, n_{\eta}\right\rangle, \quad n_{\theta}, n_{\eta} \in\{0,1,2, \ldots\}
$$

where $n_{\theta}, n_{\eta}$ enumerate the $\theta$ and $\eta$ particles present. We assume that in a dilute system $(T \ll M)$ the observables $n, \chi$ are dominated by three sectors of the Fock space, namely

\footnotetext{
${ }^{2}$ This omission, also made in ref. [10, corresponds to the assumption that $n$-body bound states of the heavy particles (here $n \geq 3$ ) have binding energies much smaller than $M$, so that they carry a minor fraction of the total dark matter number density and do not substantially contribute to the pair annihilation process. This should be well justified for $M \sim \mathrm{TeV} \gg \mathrm{GeV}$.
} 
$\left(n_{\theta}, n_{\eta}\right)=(1,0),(0,1),(1,1)$, whereas the contributions of the sectors $\left(n_{\theta}, n_{\eta}\right)=(2,0),(0,2)$ and those of any three-particle and higher states are Boltzmann-suppressed. As this simplifies formal manipulations, we stay in a finite volume for a moment, so that one-particle states are parametrized by a set of discrete momenta $\left\{\mathbf{p}_{\theta}\right\}$, with the corresponding degeneracies $c_{\theta}=c_{\eta}=d_{s} N_{\mathrm{c}}$. The two-particle states can be either bound or scattering states; for brevity, we use a scattering-like notation here, parametrizing the states with a pair of momenta $\left(\mathbf{p}_{\theta}, \mathbf{p}_{\eta}\right)$, and denoting by $E_{p_{\theta}, p_{\eta}}$ the corresponding energy and by $c_{\theta, \eta}$ the degeneracy factor. With this notation, and sticking to a state normalization without volume factors in order to avoid clutter, the number density of eq. (2.4) can schematically be evaluated as

$$
n \simeq \frac{1}{V} \frac{\sum_{\mathbf{p}_{\theta}} c_{\theta} e^{\beta\left(\mu-E_{p_{\theta}}\right)}+\sum_{\mathbf{p}_{\eta}} c_{\eta} e^{\beta\left(\mu-E_{p_{\eta}}\right)}+2 \sum_{\mathbf{p}_{\theta}, \mathbf{p}_{\eta}} c_{\theta, \eta} e^{\beta\left(2 \mu-E_{p_{\theta}, p_{\eta}}\right)}}{1+\sum_{\mathbf{p}_{\theta}} c_{\theta} e^{\beta\left(\mu-E_{p_{\theta}}\right)}+\sum_{\mathbf{p}_{\eta}} c_{\eta} e^{\beta\left(\mu-E_{p_{\eta}}\right)}} .
$$

The factor 2 in the third term of the numerator emerges because there are two particles in the sector $n_{\theta}=n_{\eta}=1$. The denominator represents normalization by $Z$ (cf. eq. (2.3)); the first term originates from the sector $n_{\theta}=n_{\eta}=0$. Since we need to go up to second order in the fugacity expansion, we need to include the next terms as well. Eq. (3.2) represents a relation between $n$ and $\mu$, and is as such a variant of the Saha equation, even if the Saha equation is usually used in a different way 3

Expanding the denominator of eq. (3.2) in the fugacity expansion and identifying the contributions from the chosen sectors of the Fock space in eq. (2.7), we find

$$
\begin{aligned}
& p_{1}=\frac{T}{V}\left[\sum_{\mathbf{p}_{\theta}} c_{\theta} e^{-\beta E_{p_{\theta}}}+\sum_{\mathbf{p}_{\eta}} c_{\eta} e^{-\beta E_{p_{\eta}}}\right], \\
& p_{2}=\frac{T}{V} \sum_{\mathbf{p}_{\theta}, \mathbf{p}_{\eta}}\left[c_{\theta, \eta} e^{-\beta E_{p_{\theta}, p_{\eta}}}-c_{\theta} c_{\eta} e^{-\beta\left(E_{p_{\theta}}+E_{p_{\eta}}\right)}\right] .
\end{aligned}
$$

For a perturbative evaluation, we write

$$
E_{p} \equiv M_{\text {rest }}+\frac{p^{2}}{2 M_{\text {kin }}}
$$

where the Salpeter correction (i.e. thermal shift of rest mass) has been included in $M_{\text {rest }}$, and $M_{\text {kin }}$ may similarly contain thermal effects. We note that, in the non-interacting limit, $E_{p_{\theta}, p_{\eta}}=E_{p_{\theta}}+E_{p_{\eta}}$ and $c_{\theta, \eta}=c_{\theta} c_{\eta}$. Then $p_{2}=0$, whereas $n_{\text {eq }}$ from eq. (2.2) becomes (for $V \rightarrow \infty)$

$$
n_{\mathrm{eq}} \approx 2 d_{s} N_{\mathrm{c}} \int_{\mathbf{p}} e^{-\beta E_{p}}
$$

\footnotetext{
${ }^{3}$ Normally one eliminates $e^{\beta \mu}$ in favour of the number densities of unbound states $\left(n_{\theta}\right.$ and $\left.n_{\eta}\right)$, viz. $e^{\beta \mu}=$ $\frac{n_{\theta}}{c_{\theta}}\left(\frac{2 \pi}{M_{\theta} T}\right)^{\frac{3}{2}} e^{\beta M_{\theta}}$. Then the last term in the numerator of eq. (3.2) is proportional to $n_{\theta} n_{\eta} e^{\beta \Delta E}$, where $\Delta E$ is the binding energy and we assume the existence of one bound state. Subsequently, if the relation of $n_{\theta}$ and $n_{\eta}$ is known (in our case $n_{\theta}=n_{\eta}$ ), they can be solved for as a function of the total number density $n$ and the exponential factor $e^{\beta \Delta E}$. Here we instead want to solve for $e^{\beta \mu}$, cf. eq. (2.12), as this is needed in eq. (1.2).
} 
Proceeding to $\hat{p}_{2}$, we may follow the argument of ref. [10] according to which eq. (3.4) should be dominated by bound states at low temperatures. Let us write

$$
E_{p_{\theta}, p_{\eta}}=2 M_{\text {rest }}+\frac{k^{2}}{4 M_{\text {kin }}}+E^{\prime},
$$

where $\mathbf{k}=\mathbf{p}_{\theta}+\mathbf{p}_{\eta}$ is the momentum of the center-of-mass motion, $k \equiv|\mathbf{k}|$, and $E^{\prime}$ is the relative energy. We may now write $\sum_{\mathbf{p}_{\theta}, \mathbf{p}_{\eta}}=\sum_{\mathbf{k}, E^{\prime}}$, and for the sum over $\mathbf{k}$ go over to infinite volume. Furthermore, it is convenient to normalize $p_{2}$ as in eq. (2.11). Thereby

$$
\hat{p}_{2}=\frac{\frac{1}{V} \sum_{\mathbf{p}_{\theta}, \mathbf{p}_{\eta}}\left[c_{\theta, \eta} e^{-\beta E_{p_{\theta}, p_{\eta}}}-c_{\theta} c_{\eta} e^{-\beta\left(E_{p_{\theta}}+E_{p_{\eta}}\right)}\right]}{\left[\frac{1}{V}\left(\sum_{\mathbf{p}_{\theta}} c_{\theta} e^{-\beta E_{p_{\theta}}}+\sum_{\mathbf{p}_{\eta}} c_{\eta} e^{-\beta E_{p_{\eta}}}\right)\right]^{2}} \simeq 2\left(\frac{\pi}{M_{\mathrm{kin}} T}\right)^{3 / 2} \sum_{-E^{\prime} \gg T} \frac{c_{\theta, \eta}}{c_{\theta}^{2}} e^{-\beta E^{\prime}} .
$$

Assuming that the contribution of the $d_{s}^{2}\left(N_{\mathrm{c}}^{2}-1\right)$ octet degrees of freedom is exponentially suppressed, and omitting any hyperfine splitting, we can set $c_{\theta, \eta} \rightarrow d_{s}^{2}$. If we furthermore assume that one bound state dominates, with the binding energy by $\Delta E \simeq \alpha^{2} M_{\text {kin }} / 4$, and require a qualitatively correct limiting behaviour on the high-temperature side, we may set

$$
T^{3} \hat{p}_{2} \simeq \frac{2}{N_{\mathrm{c}}^{2}}\left(\frac{\pi T}{M_{\text {kin }}}\right)^{3 / 2}\left(e^{\beta \Delta E}-1\right)
$$

We stress that this result should only be interpreted as an order-of-magnitude estimate, and that it is exponentially sensitive to the choice of the value of $\alpha$ in $\Delta E$.

\section{Non-perturbative formulation}

To go further, it is helpful to give an imaginary-time path-integral representation to the observables in eqs. (2.4) and (2.5). As we assume the fields $\theta, \eta$ to be non-relativistic, they propagate in one time direction only, and their propagators are discontinuous across the imaginary-time interval. Therefore some care is needed for defining a proper time ordering.

For $n$, a convenient possibility is to split the time arguments by an infinitesimal amount, $n=\left\langle\theta^{\dagger}(0, \mathbf{0}) \theta\left(0^{-}, \mathbf{0}\right)+\eta^{\dagger}(0, \mathbf{0}) \eta\left(0^{-}, \mathbf{0}\right)\right\rangle$. Antiperiodicity implies $\theta\left(0^{-}, \mathbf{0}\right)=-\theta(\beta, \mathbf{0})$, and we can subsequently use the Grassmann nature of the fields to anticommute $\theta(\beta, \mathbf{0})$ to the left. Therefore,

$$
n=\operatorname{Tr}\left\langle\theta(\beta, \mathbf{0}) \theta^{\dagger}(0, \mathbf{0})+\eta(\beta, \mathbf{0}) \eta^{\dagger}(0, \mathbf{0})\right\rangle .
$$

We now denote (cf. appendix A of ref. [9])

$$
\left\langle\theta(\beta, \mathbf{x}) \theta^{\dagger}(0, \mathbf{x})\right\rangle_{0} \equiv e^{\beta \mu} G_{\mathbf{x}}, \quad\left\langle\eta(\beta, \mathbf{x}) \eta^{\dagger}(0, \mathbf{x})\right\rangle_{0}=e^{\beta \mu} G_{\mathbf{x}}^{*},
$$

where $\langle\ldots\rangle_{0}$ denotes a contraction of the Grassmann fields. Gauge fields are left to be averaged over later on, which is denoted by $\langle\ldots\rangle$. Then eq. (4.1) becomes

$$
n=2 e^{\beta \mu}\left\langle\operatorname{Re} \operatorname{Tr} G_{\mathbf{0}}\right\rangle .
$$


For $\chi$, we point-split each $n$, and in addition make use of the fact that $\int_{\mathbf{x}} n(\tau, \mathbf{x})$ is a conserved charge, whereby we can set the two $n$-operators at different times. So,

$$
\chi=\int_{\mathbf{x}}\left\langle\left[\theta^{\dagger}(\tau, \mathbf{x}) \theta\left(\tau^{-}, \mathbf{x}\right)+\eta^{\dagger}(\tau, \mathbf{x}) \eta\left(\tau^{-}, \mathbf{x}\right)\right]\left[\theta^{\dagger}(0, \mathbf{0}) \theta\left(0^{-}, \mathbf{0}\right)+\eta^{\dagger}(0, \mathbf{0}) \eta\left(0^{-}, \mathbf{0}\right)\right]-n^{2}\right\rangle .
$$

Subsequently we can replace $\theta\left(0^{-}, \mathbf{0}\right)$ through $-\theta(\beta, \mathbf{0})$, and again anticommute fields. This leads to

$$
\chi=\int_{\mathbf{x}}\left\langle 4 e^{2 \beta \mu} \operatorname{Re} \operatorname{Tr} G_{\mathbf{x}} \operatorname{Re} \operatorname{Tr} G_{\mathbf{0}}+2 e^{\beta \mu} \operatorname{Re} \operatorname{Tr} G_{\mathbf{0}}-n^{2}\right\rangle,
$$

where the middle term originates from contractions like

$$
\delta \chi=\int_{\mathbf{x}}\left\langle\theta(\beta, \mathbf{0}) \theta^{\dagger}(\tau, \mathbf{x})\right\rangle_{0}\left\langle\theta(\tau, \mathbf{x}) \theta^{\dagger}(0, \mathbf{0})\right\rangle_{0}
$$

after making use of the semigroup property of the propagator 4

Now we can subtract $n$ of eq. (4.3) from $\chi$ of eq. (4.5) according to eq. (2.10), thus obtaining a representation for $p_{2}$. Moreover, normalizing according to eq. (2.11), where $n_{\text {eq }}=2\left\langle\operatorname{Re} \operatorname{Tr} G_{\mathbf{0}}\right\rangle$ according to eqs. (2.2) and (4.3), we find

$$
\hat{p}_{2}=\frac{\int_{\mathbf{x}}\left\{\left\langle\operatorname{Re} \operatorname{Tr} G_{\mathbf{x}} \operatorname{Re} \operatorname{Tr} G_{\mathbf{0}}\right\rangle-\left\langle\operatorname{Re} \operatorname{Tr} G_{\mathbf{0}}\right\rangle^{2}\right\}}{2\left\langle\operatorname{Re} \operatorname{Tr} G_{\mathbf{0}}\right\rangle^{2}} .
$$

The numerator represents a "disconnected" contraction, with two heavy particle propagators not cancelling each other only because they are connected by gauge field lines.

\section{Lattice measurement}

In order to obtain non-perturbative information on the influence of bound states, we have measured $\hat{p}_{2}$ from eq. (4.7) with methods of non-relativistic lattice QCD. We have considered spinors with $s=\frac{1}{2}$, however we expect spin effects to be very small so that the results also apply to $s=0$. For a good statistical precision, it is helpful to make use of translational invariance and rephrase the measurement of eq. (4.7) in analogy with eq. (2.4),

$$
T^{3} \hat{p}_{2}=\lim _{V \rightarrow \infty} \frac{T^{3} V}{2} \frac{\left\langle\mathcal{G}^{2}\right\rangle-\langle\mathcal{G}\rangle^{2}}{\langle\mathcal{G}\rangle^{2}}, \quad \mathcal{G} \equiv \frac{1}{V} \int_{\mathbf{x}} \operatorname{Re} \operatorname{Tr} G_{\mathbf{x}} .
$$

\footnotetext{
${ }^{4}$ In a Dirac notation, this corresponds to the use of a completeness relation,

$$
\int_{\mathbf{x}}\langle\beta, \mathbf{0} \mid \tau, \mathbf{x}\rangle\langle\tau, \mathbf{x} \mid 0, \mathbf{0}\rangle=\langle\beta, \mathbf{0} \mid 0, \mathbf{0}\rangle, \quad 0<\tau<\beta .
$$
}




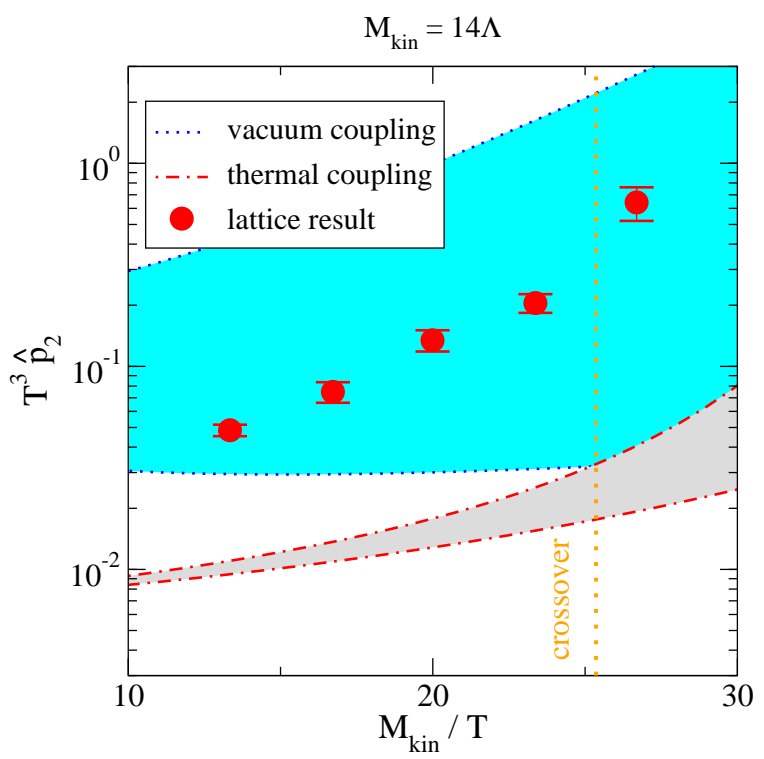

Figure 1: Comparison of an order-of-magnitude estimate of $T^{3} \hat{p}_{2}$ from sec. B and a lattice estimate from sec. 5] The dashed line shows a temperature at which confinement sets in. The errors of the lattice results are statistical only; systematic uncertainties could be as large as $\sim 50 \%$. In any case, based on this test, a vacuum-like coupling performs best at low temperatures, whereas towards high temperatures the slope seen in the data agrees better with a thermal scale choice (i.e. $\bar{\mu} \sim \pi T$ ).

The propagator $G_{\mathbf{x}}$ is constructed as explained in ref. [9] 5

On a lattice, $T=1 /\left(N_{\tau} a_{\tau}\right)$ and $V=\left(N_{s} a_{s}\right)^{3}$, where $a_{\tau}, a_{s}$ are the temporal and spatial lattice spacings and $N_{\tau}, N_{s}$ are the numbers of lattice points in these directions, respectively. The details of the lattice setup were summarized in ref. [13]; we have relied on refs. [14 17] for the adjustment of the bare parameters as well as for the generation of the gauge configurations, both of which carry a substantial numerical cost.

The key idea of the lattice test is that the importance of any effects associated with bound states depends on the ratio $\Delta E / T \sim \alpha^{2} M / T$, where $\Delta E$ is the binding energy and $M$ is the dark matter mass scale. In the following, we denote by $\Lambda$ the $\overline{\mathrm{MS}}$ scale parameter. In cosmological applications, the phenomenologically relevant mass scale is $M \gtrsim 1 \mathrm{TeV}$ $\gg 10^{3} \Lambda$, and correspondingly the coupling $\alpha \sim 0.1$ is "small". In this situation boundstate effects are expected to be large only in the regime $M / T \gtrsim 1 / \alpha^{2} \sim 100$. In contrast, lattice simulations are best suited to moderate temperatures, $T / \Lambda \sim 0.5 \ldots 1.0$, and a situation without large scale hierarchies. Then the coupling is "large", $\alpha \gtrsim 0.3$, and bound-state effects are important already for $M / T \sim 10 \ldots 30$. The idea is now that if we can use lattice to scrutinize analytic estimates in the domain of large couplings, we should be confident that

\footnotetext{
${ }^{5}$ For $M / T \rightarrow \infty$, the numerator and denominator of eq. (5.1) correspond to the Polyakov loop susceptibility and expectation value squared, respectively.
} 
they apply in the cosmological domain of small couplings.

The results of the lattice measurements are shown in fig. 1, where they are also compared with the order-of-magnitude estimate from sec. 3. The dominant uncertainty of the latter is the choice of $\alpha$. By a "vacuum coupling" we indicate that $\alpha$ has been evaluated at a scale $(0.5 \ldots 2.0) e^{-\gamma_{\mathrm{E}}} / a$, where $a=2 /(M \alpha)$ is the Bohr radius; the factor $e^{-\gamma_{\mathrm{E}}}$ is inspired by refs. [18,19]; and we have solved the implicit equation for $\alpha$ numerically, by employing 2-loop running. By a "thermal coupling" we indicate the dimensionally reduced value, as specified in appendix A of ref. [25].

In view of the experience from ref. [13], where other observables were measured in the same temperature range, as well as the exponential dependence on $\alpha$, the rough qualitative agreement between the lattice and analytic results seen in fig. 1 should be considered reasonable. The lesson we draw is that at low temperatures the vacuum coupling should be a fair choice, whereas at high temperatures, where bound states are less prominent and ultimately dissolve, the results tend gradually towards a thermal value (though they do not reach it within the domain of large $\alpha$ ). In sec. [6 we interpolate between these two possibilities.

\section{A dark matter application}

Having tested $\hat{p}_{2}$ from eq. (3.9) against lattice data in sec. 5, we are now ready to apply the same estimate to a simple but realistic cosmological computation. In this case we add a neutral field to the model (as dark matter proper), and let the charged field (mediator) be in general heavier, by an amount $\Delta M$.

Specifically, we consider the setup reviewed in refs. [20,21] and recently studied for boundstate effects in refs. [22 28], in which the dark matter particle is a singlet Majorana fermion, and the dark sector also contains a strongly coupled scalar mediator, a "stop". If the stop is not much heavier than the Majorana fermion, strong interactions between a stop and antistop open up a very efficient annihilation channel in the early universe, reducing the dark matter abundance to an acceptable level even in the multi-TeV mass range. Simultaneously, the $p$-wave suppressed annihilations of the Majorana fermion at low energies guarantee that constraints from indirect detection can be satisfied. The direct detection constraints are weak, if the Yukawa interaction couples the stop dominantly to 3rd generation quarks [27]. Furthermore collider constraints can be evaded if the stops are heavier than $\sim 1 \mathrm{TeV}$.

The same model was studied within the current formalism in refs. [25, 27, however under the assumption $\hat{p}_{2}=0$, whereby $e^{\beta \mu} n_{\text {eq }}=n$ according to eq. (2.12). This led to the problem that bound-state effects became extremely large at $z \equiv M / T \gg 10^{3}$. In order to avoid this problem, the mass splitting $\Delta M$ was chosen large enough to satisfy $2 \Delta M>\Delta E$, so that bound states of stops were always heavier than scattering states of Majorana fermions, and thus ultimately exponentially suppressed. The equations were integrated down to $z=10^{3}$. 


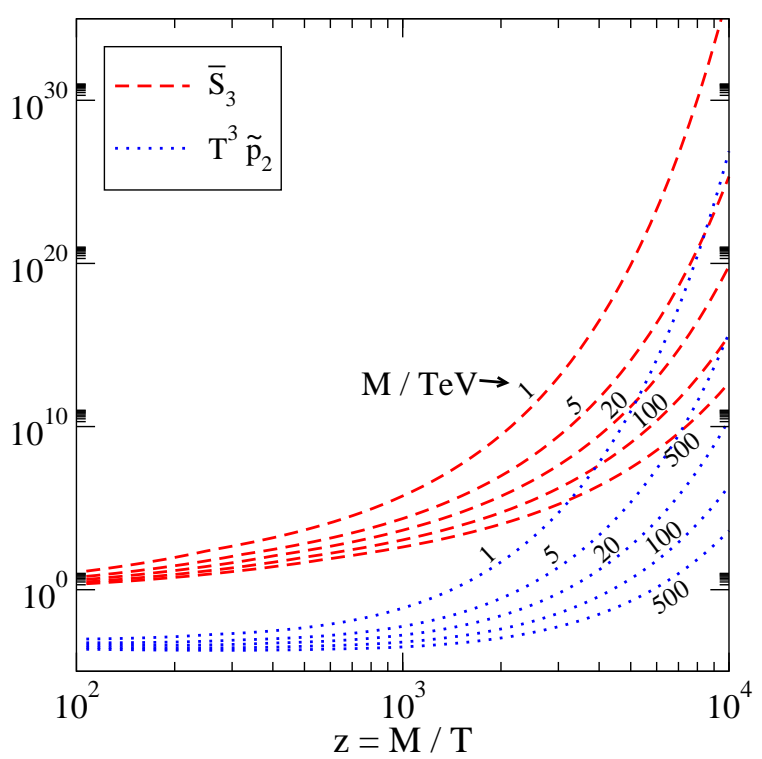

Figure 2: Values of the thermally averaged Sommerfeld factor $\bar{S}_{3}$ (cf. eq. (6.3)) and the rescaled susceptibility $T^{3} \tilde{p}_{2}$ (cf. eq. (6.2)) for $M=1 \ldots 500 \mathrm{TeV}$.

We have now included $\hat{p}_{2}$ in the dynamics described by eq. (1.2), by solving for $e^{\beta \mu} n_{\text {eq }}$ from eq. (2.12). The presence of the neutral field implies that

$$
n_{\mathrm{eq}} \simeq 2\left(\frac{M T}{2 \pi}\right)^{\frac{3}{2}} e^{-\beta M}\left(1+N_{\mathrm{c}} e^{-\beta \Delta M_{T}}\right)
$$

where the thermally modified mass difference $\Delta M_{T}$ is given in eq. (4.8) of ref. [25], and we have dropped the subscript from $M_{\text {kin }}$ for simplicity. Given the normalization by $n_{\text {eq }}^{2}$ (cf. eq. (2.11) ), the order-of-magnitude estimate from eq. (3.9) becomes

$$
\hat{p}_{2} \simeq \frac{N_{\mathrm{c}}^{2}}{\left(N_{\mathrm{c}}+e^{\left.\beta \Delta M_{T}\right)^{2}}\right.} \tilde{p}_{2}, \quad T^{3} \tilde{p}_{2} \equiv \frac{2}{N_{\mathrm{c}}^{2}}\left(\frac{\pi T}{M}\right)^{3 / 2}\left(e^{\beta \Delta E}-1\right),
$$

where $\Delta E=\alpha^{2} M / 4$, and we have for convenience defined a $\Delta M_{T}$-independent $\tilde{p}_{2}$. The corresponding approximation for the attractive Sommerfeld factor reads [25]

$$
\bar{S}_{3} \approx\left(\frac{4 \pi}{M T}\right)^{\frac{3}{2}} \frac{e^{\beta \Delta E}}{\pi a^{3}}
$$

where $a=2 /(M \alpha)$ is the Bohr radius. Inspired by the tests in sec. 5, at low temperatures the coupling $\alpha$ is evaluated at the $\overline{\mathrm{MS}}$ scale $\sim e^{-\gamma_{\mathrm{E}}} / a$, and at high temperatures we use a thermal coupling; the crossover takes place at $z \approx 250 \ldots 600$ for $M=1 \ldots 500 \mathrm{TeV}$. This $\bar{S}_{3}$ attaches rather smoothly to the more elaborate results described in ref. [25]; in practice we 


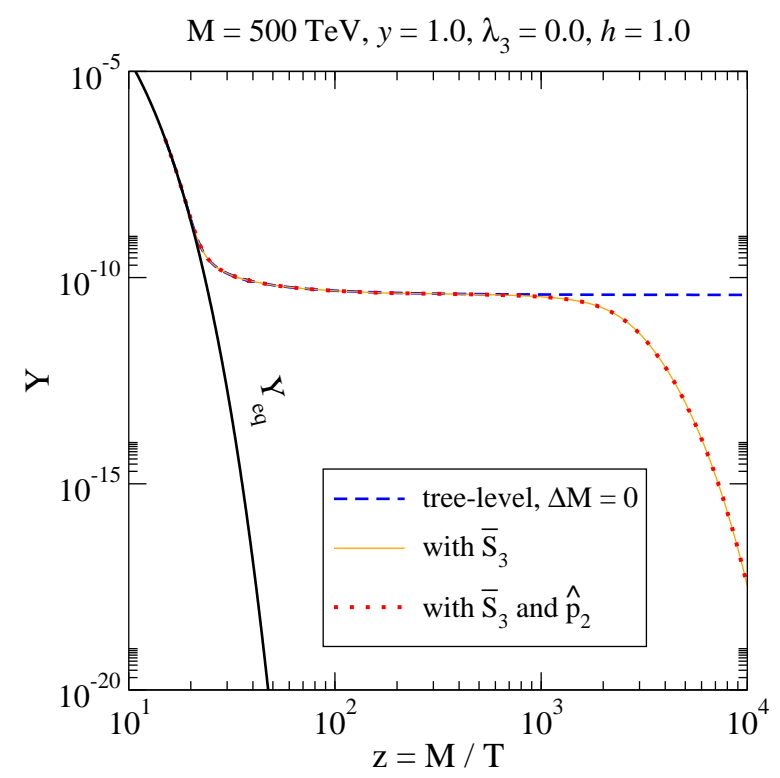

Figure 3: Examples of a solution, for $M=500 \mathrm{TeV}$, with tree-level annihilation rates ("tree-level") and after including a thermally averaged Sommerfeld factor ("with $\bar{S}_{3}$ ") and a susceptibility ("with $\bar{S}_{3}$ and $\left.\hat{p}_{2}{ }^{\prime \prime}\right)$. The symbols $y, \lambda_{3}, h$ refer to couplings defined in ref. 25], whose precise values have little impact on the general pattern. This plot assumes that kinetic/ionization equilibrium is maintained in the dark sector.

can use the simplified expression from eq. (6.3) at $z \gtrsim 200$. The repulsive Sommerfeld factors $\bar{S}_{4,5}[25$, which are not important at late times, are frozen to their values at $z \simeq 200$.

Numerical values of $T^{3} \tilde{p}_{2}$ and $\bar{S}_{3}$ are shown in fig. 2. As anticipated in ref. [10], the presence of a non-zero $\hat{p}_{2}$ in principle "regulates" the late-time behaviour of the system: the growth of $\langle\sigma v\rangle$, which is proportional to $\bar{S}_{3}$, is compensated for by the growth of $\hat{p}_{2}$, which appears in the numerator (cf. eqs. (1.2) and (2.12)). Alas, we find that in practice this regulation is not efficient in this model. This can be understood by inspecting the combination

$$
8 \hat{p}_{2} n=8 T^{3} \hat{p}_{2}\left(\frac{s}{T^{3}}\right) Y
$$

that appears in eq. (2.12) (here $Y \equiv n / s$ ). The entropy density is $s / T^{3} \lesssim 50$. Recalling

$$
\Omega_{\mathrm{dm}} h^{2} \approx \frac{Y\left(z_{\mathrm{final}}\right) M}{\left[3.645 \times 10^{-12} \mathrm{TeV}\right]} \approx 0.12,
$$

we are interested in yields $Y \simeq 10^{-13}$. According to eq. (6.4), we would then need $T^{3} \hat{p}_{2} \gg$ $10^{11}$, in order to have $8 \hat{p}_{2} n \gg 1$ and thus a substantial regularization through $\hat{p}_{2}$.

Now, according to fig. 2 , values $T^{3} \hat{p}_{2} \gtrsim 10^{11}$ can indeed be found if $M \lesssim 20 \mathrm{TeV}$, however they only set in at large $z$. Unfortunately, by this time $\bar{S}_{3} \gg 10^{10}$, whereby $Y \simeq 10^{-13}$ can actually not be found in this model. The situation is illustrated in fig. 3 for an extreme case 
$M=500 \mathrm{TeV}$, chosen to push the initial $Y$ as large as possible. It is clear that the large $\bar{S}_{3}$ rapidly pulls $Y$ down to such small values that the increasing $\hat{p}_{2}$ has no visible effect. To summarize, we find no stabilizing effect from $\hat{p}_{2}$ in the whole mass range considered (1...500 TeV), leaving $\Delta M>\Delta E / 2$ as the only possible (equilibrium) regulator.

\section{Conclusions}

The purpose of this paper has been to explore the implications of the modified Lee-Weinberg equation (cf. eq. (1.2) ) put forward in ref. [10]. On one hand, we have shown how the coefficient $\hat{p}_{2}$, which captures the essence of the Saha equation (cf. eq. (2.12)), can be related to a "susceptibility" (cf. eq. (5.1)), which can be measured non-perturbatively within a nonrelativistic lattice QCD framework (cf. fig. 1). On the other hand, we have shown how $\hat{p}_{2}$ can be used in a practical dark matter computation, where it implements ionization equilibrium in accordance with the Saha equation, and therefore guarantees that bound states appear with their thermal abundance (this assumption ceases to be valid at very low temperatures). As proposed in ref. [10], the presence of $\hat{p}_{2}$ can in principle regulate the late-time behaviour of the system, in addition to the regularization provided by an explicit mass difference $\Delta M$ in the dark sector, or the non-equilibrium effects that inevitably take over at the very end.

However, considering a concrete model with a strongly interacting mediator, we find that in practice the regularization by $\hat{p}_{2}$ is insufficient to make the system viable if $2 \Delta M<\Delta E$, where $\Delta E$ is the binding energy for bound states in the mediator sector (cf. fig. 3 and the discussion around eq. (6.4)). This implies that the viable domain remains sensitive to the value of $\Delta M$ in this model (nevertheless the viable domain extends at least to the multi-TeV range as discussed in refs. [25, 27]). Whether other models could behave differently is not

clear at the moment, even if we note that in general $\bar{S}_{3} /\left(T^{3} \hat{p}_{2}\right) \simeq(M \alpha / T)^{3} \gg 1$ at low temperatures, suggesting that $\hat{p}_{2}$ is not sufficient to compensate for the effect of $\bar{S}_{3}$.

It is perhaps prudent to stress that our current analytic values of $\hat{p}_{2}$ amount just to an orderof-magnitude estime, originating from a Coulomb-like ground-state binding energy. At least on the high-temperature side, this could in principle be promoted into a consistent leadingorder perturbative computation, however this is demanding, given that eq. (4.7) originates from a disconnected contraction, and is therefore of 3-loop order, i.e. $\mathcal{O}\left(\alpha^{2}\right)$.

\section{Acknowledgements}

We thank the FASTSUM collaboration for providing the unquenched gauge configurations used in our lattice measurements. S.B. thanks AEC/ITP of the University of Bern for hospitality during initial stages of this work. S.K. was supported by the National Research 
Foundation of Korea under grant No. 2018R1A2A2A05018231 funded by the Korean government (MEST) and in part by NRF-2008-000458. M.L. was supported by the Swiss National Science Foundation (SNF) under grant 200020-168988.

\section{References}

[1] B.W. Lee and S. Weinberg, Cosmological Lower Bound on Heavy Neutrino Masses, Phys. Rev. Lett. 39 (1977) 165.

[2] J. Bernstein, L.S. Brown and G. Feinberg, The Cosmological Heavy Neutrino Problem Revisited, Phys. Rev. D 32 (1985) 3261.

[3] K. Griest and D. Seckel, Three exceptions in the calculation of relic abundances, Phys. Rev. D 43 (1991) 3191.

[4] W. Detmold, M. McCullough and A. Pochinsky, Dark Nuclei I: Cosmology and Indirect Detection, Phys. Rev. D 90 (2014) 115013 [1406.2276].

[5] B. von Harling and K. Petraki, Bound-state formation for thermal relic dark matter and unitarity, JCAP 12 (2014) 033 [1407.7874].

[6] F. Karsch, D. Kharzeev and H. Satz, Sequential charmonium dissociation, Phys. Lett. B 637 (2006) 75 hep-ph/0512239.

[7] D. Bödeker and M. Laine, Heavy quark chemical equilibration rate as a transport coefficient, JHEP 07 (2012) 130 [1205.4987].

[8] G.T. Bodwin, E. Braaten and G.P. Lepage, Rigorous QCD analysis of inclusive annihilation and production of heavy quarkonium, Phys. Rev. D 51 (1995) 1125; ibid. 55 (1997) 5853 (E) hep-ph/9407339.

[9] S. Kim and M. Laine, Rapid thermal co-annihilation through bound states in QCD, JHEP 07 (2016) 143 [1602.08105].

[10] T. Binder, L. Covi and K. Mukaida, Dark Matter Sommerfeld-enhanced annihilation and Boundstate decay at finite temperature, Phys. Rev. D 98 (2018) 115023 [1808.06472].

[11] D. Bödeker and M. Laine, Kubo relations and radiative corrections for lepton number washout, JCAP 05 (2014) 041 [1403.2755].

[12] D. Bödeker and M. Sangel, Order $g^{2}$ susceptibilities in the symmetric phase of the Standard Model, JCAP 04 (2015) 040 [1501.03151].

[13] S. Kim and M. Laine, Studies of a thermally averaged p-wave Sommerfeld factor, Phys. Lett. B 795 (2019) 469 [1904.07882].

[14] R.G. Edwards, B. Joo and H.W. Lin, Tuning for Three-flavors of Anisotropic Clover Fermions with Stout-link Smearing, Phys. Rev. D 78 (2008) 054501 [0803.3960]. 
[15] H.W. Lin et al. [Hadron Spectrum Collaboration], First results from $2+1$ dynamical quark flavors on an anisotropic lattice: Light-hadron spectroscopy and setting the strange-quark mass, Phys. Rev. D 79 (2009) 034502 [0810.3588].

[16] C. Allton et al., 2+1 flavour thermal studies on an anisotropic lattice, PoS LATTICE 2013 (2014) 151 [1401.2116].

[17] G. Aarts et al, The bottomonium spectrum at finite temperature from $N_{f}=2+1$ lattice $Q C D$, JHEP 07 (2014) 097 [1402.6210].

[18] Y. Schröder, The Static potential in QCD to two loops, Phys. Lett. B 447 (1999) 321 hep-ph/9812205.

[19] R.N. Lee, A.V. Smirnov, V.A. Smirnov and M. Steinhauser, Analytic three-loop static potential, Phys. Rev. D 94 (2016) 054029 [1608.02603].

[20] M. Garny, A. Ibarra and S. Vogl, Signatures of Majorana dark matter with t-channel mediators, Int. J. Mod. Phys. D 24 (2015) 1530019 [1503.01500].

[21] M. Garny, J. Heisig, M. Hufnagel and B. Lülf, Top-philic dark matter within and beyond the WIMP paradigm, Phys. Rev. D 97 (2018) 075002 [1802.00814].

[22] S.P. Liew and F. Luo, Effects of QCD bound states on dark matter relic abundance, JHEP 02 (2017) 091 [1611.08133].

[23] A. Mitridate, M. Redi, J. Smirnov and A. Strumia, Cosmological Implications of Dark Matter Bound States, JCAP 05 (2017) 006 [1702.01141].

[24] W.Y. Keung, I. Low and Y. Zhang, A Reappraisal on Dark Matter Co-annihilating with a Top/Bottom Partner, Phys. Rev. D 96 (2017) 015008 [1703.02977].

[25] S. Biondini and M. Laine, Thermal dark matter co-annihilating with a strongly interacting scalar, JHEP 04 (2018) 072 [1801.05821].

[26] J. Harz and K. Petraki, Radiative bound-state formation in unbroken perturbative non-Abelian theories and implications for dark matter, JHEP 07 (2018) 096 [1805.01200].

[27] S. Biondini and S. Vogl, Coloured coannihilations: Dark matter phenomenology meets nonrelativistic EFTs, JHEP 02 (2019) 016 [1811.02581].

[28] H. Fukuda, F. Luo and S. Shirai, How Heavy can Neutralino Dark Matter be?, JHEP 04 (2019) 107 [1812.02066]. 